\title{
Tendinopatías por fluoroquinolonas
}

\author{
M. MEDRANO SAN ILDEFONSO, J. A. MAURI LLERDA ${ }^{1}$, C. BRUSCAS IZU \\ Sección de Reumatología. Hospital Universitario Miguel Servet. ${ }^{I}$ Servicio de Neurología. \\ Hospital Clínico Universitario. Zaragoza
}

\author{
FLUOROQUINOLONE-INDUCED TENDON DISEASES
}

\begin{abstract}
RESUMEN
Las tendinitis y roturas tendinosas inducidas por fluoroquinolonas, aunque infrecuentes, han sido documentadas en la literatura desde 1983. Presentamos cinco casos de temdinitis inducidas por fluoroquinolonas, tres por levofloxacino y dos por ciprofloxacino. Revisamos los conocimientos actuales de esta asociación e insistimos en la importancia de la detección precoz para evitar roturas tendinosas.
\end{abstract}

PALABRAS CLAVE: Fluoroquinolonas. Tendinitis. Tendinopatía. Rotura tendinosa.

\begin{abstract}
Tendinitis and tendon ruptures induced by fluoroquinolones, while uncommon, have beeb documented in the literature since 1983. We report five cases of tendinitis induced by fluoroquinolones, three caused by levofloxacin and two by ciprofloxacin. We revise actual knowledge of this association and we insist on how important is an early detection to prevent tendon rupture.
\end{abstract}

KEY WORDS: Fluoroquinolones. Tendinitis. Tendinophaty. Tendon rupture.

\section{INTRODUCCIÓN}

Las quinolonas son una familia de antibióticos de amplio espectro de uso común en la práctica médica. El desarrollo de las nuevas fluoroquinolonas ha mejorado su seguridad y ha aumentado su eficacia antimicrobiana. Los efectos secundarios mas frecuentes son gastrointestinales, cutáneos y neurológicos (1). Las tendinitis y roturas tendinosas son menos frecuentes (2). Presentamos cinco casos de tendinopatías inducidas por fluoroquinolonas, tres por levofloxaccino y dos por ciprofloxacino.

\section{CASOS APORTADOS}

Caso 1. Varón de 70 años de edad con antecedentes de EPOC, con frecuentes reagudizaciones que precisan de tratamiento con corticoides. Acude a urgencias por presentar fiebre alta, tos, expectoración, disnea y dolor en hemitórax derecho. En la Rx de tórax se con- firma la existencia de una neumonía en el lóbulo inferior derecho. Se instaura tratamiento con broncodilatadores, corticoides y levofloxacino $(500 \mathrm{mg} /$ día). Al tercer día de iniciar el tratamiento, presenta dolor y tumefacción de ambos tendones aquíleos, con marcada impotencia funcional. Se diagnostica de tendinitis aquílea bilateral por levofloxacino por lo que se suspende este tratamiento, se recomienda reposo y el cuadro se resuelve en dieciocho días.

Caso 2. Mujer de 67 años con antecedentes de HTA, riñón poliquístico e insuficiencia renal. Historia de infecciones urinarias previas que se han tratado con levofloxacino sin presentar efectos secundarios. En un nuevo episodio de infección urinaria, se instaura tratamiento con ciprofloxacino $(500 \mathrm{mg} / 12 \mathrm{~h})$, presentando la paciente a las 48 horas, dolor e impotencia funcional en ambos hombros. En la exploración se objetiva un arco doloroso con maniobras contrarresistencia positivas en ambos supraespinosos. En la Rx no se observan calcificaciones tendinosas ni ningún dato de interés. Se suspende el tratamiento con ciprofloxacino, quedando asintomática en diez días. Posteriormente ha precisado tratamiento con levofloxacino sin presentar reacciones adversas.

Caso 3. Varón de 73 años de edad, con antecedentes de asma, en tratamiento corticoideo, que consulta por presentar dolor y tumefac-

Trabajo aceptado: 11 de diciembre de 2006 


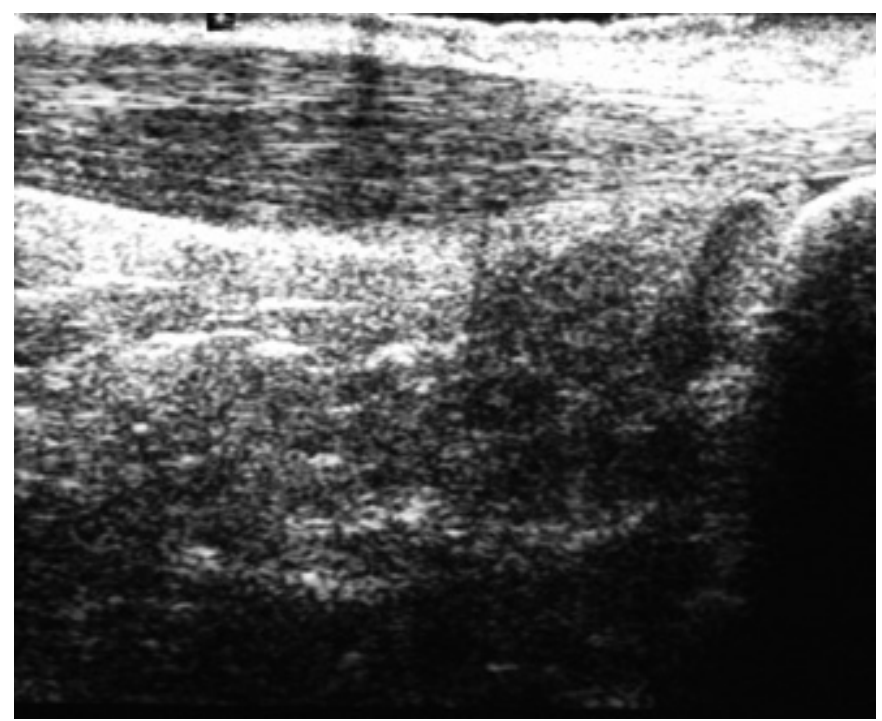

Fig. 1. Ecografía de tendón aquíleo derecho: engrosamiento del espesor del tendón aquíleo derecho en su tercio central, conservando la disposición fibrilar. Hallazgos en relación con tedinopatía sin signos de rotura.

ción en regiones aquíleas con impotencia funcional de cinco días de evolución. Los síntomas se iniciaron a las 48 horas de iniciar tratamiento con levofloxacino (500 mg/día) por una infección de vías respiratorias altas. Se realiza ecografía (Fig. 1) que demuestra un engrosamiento del espesor del tendón de Aquiles bilateral en su tercio central conservando la disposición fibrilar. Se suspende el tratamiento antibiótico y mediante reposo y AINES el cuadro se resuelve en veinte días.

Caso 4. Mujer de 68 años, con antecedentes de epilepsia y polimialgia reumática, en tratamiento con $10 \mathrm{mg}$ /día de prednisona. Presenta dolor, aumento de tamaño e impotencia funcional en aquíleo derecho a las siete días de iniciar tratamiento con levofloxacino (500 $\mathrm{mg}$ /día) indicado por una infección urinaria. Mediante Ecografía se demuestra la existencia de rotura parcial del tendón. Tras retirar el fármaco y tratamiento conservador con reposo y ortesis el cuadro se resuelve en dos meses.

Caso 5. Varón de 62 años de edad, con antecedentes de HTA y diabetes. A los diez días de iniciar tratamiento con ciprofloxacino $(500 \mathrm{mg} / 12 \mathrm{~h}$ ) indicado por un absceso perianal, comienza con dolor e impotencia funcional en el tendón de Aquiles izquierdo. A la palpación se aprecia un evidente engrosamiento del tendón. Se realiza RM (Fig. 2) que objetiva un engrosamineto fusiforme del tercio medio y superior del tendón de Aquiles izquierdo, con rotura intrasustancia, en su unión músculo-tendinosa y sin retracción. Se suspende el ciprofloxacino y mediante reposo, AINES y tratamiento rehabilitadoe se resuelve la sintomatología en dos meses.

\section{DISCUSIÓN}

La tendinitis y rotura tendinosa por fluoroquinolonas, aunque infrecuente, es un fenómeno documentado en la literatura desde 1983 (3). En esta primera descripción fue el norfloxacino el fármaco desencadenante en pacientes con insuficiencia renal. Posteriormente otras fluoroquinolonas: oxfloxacino, ciprofloxacino, levofloxacino, moxifloxacino, pefloxacino, enoxacino..., también han sido implicadas (4-19). La epidemiología no es suficientemente conocida y se estima una incidencia de 15 a 20 casos/100.000 tratamientos con estos antibióticos $(2,4)$. En

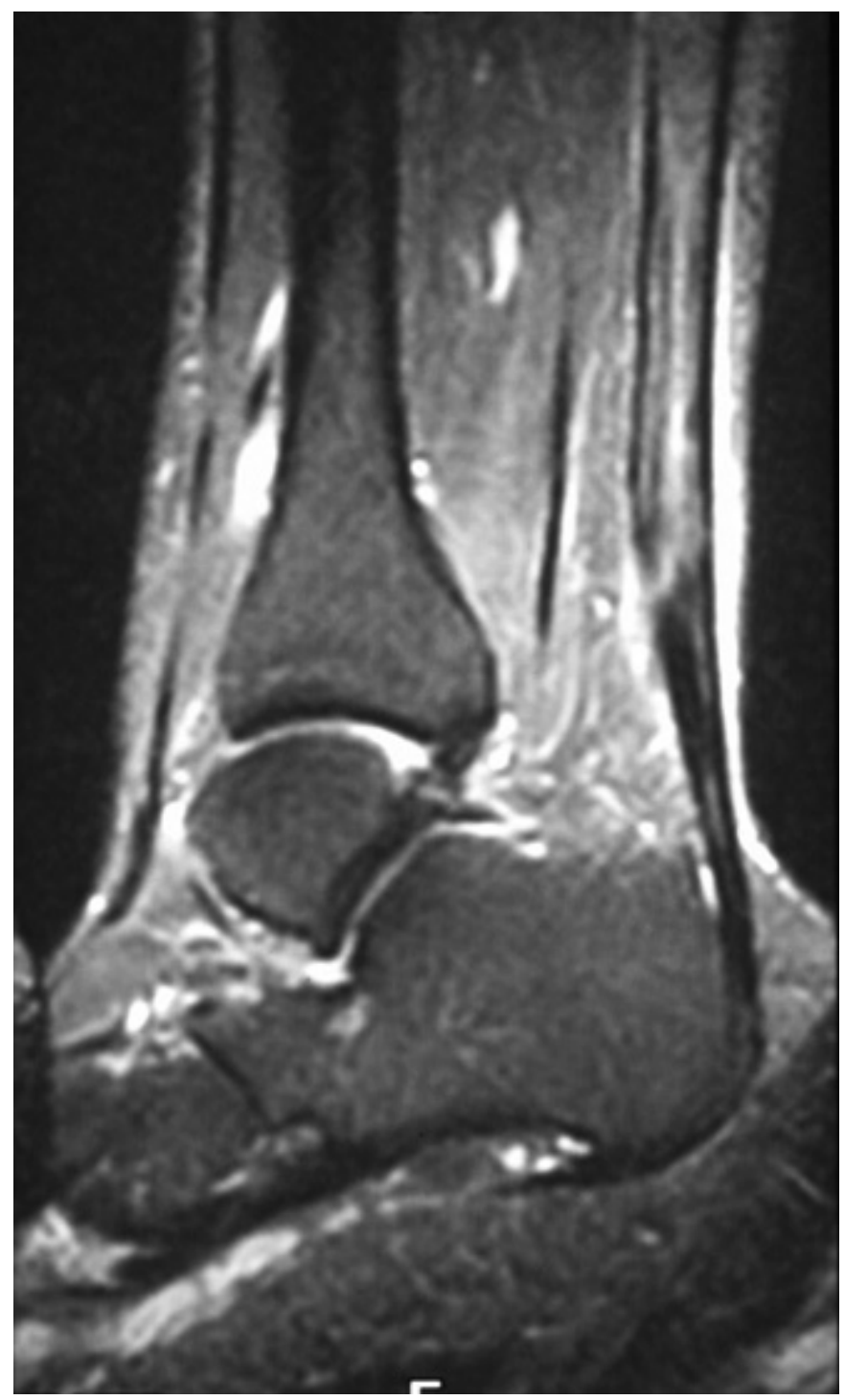

Fig. 2. RM de pie izquierdo: engrosamiento fusiforme del tercio medio y superior de Aquiles izquierdo. Rotura intrasustancia en su unión músculo-tendinosa, sin retracción.

un estudio reciente se considera que del 2 al $6 \%$ de todas las roturas del tendón de Aquiles en mayores de 60 años pueden ser atribuidas al tratamiento con estos fármacos (11). El mecanismo de producción es desconocido, se han propuesto diversas teorías: el estrés mecánico, la toxicidad directa sobre las fibras tendinosas, un mecanismo inflamatorio de características similares a la artritis por microcristales, una isquemia local y alteraciones preexistentes en el tendón. $(7,11,12)$.

El periodo de latencia entre el inicio del tratamiento y el desarrollo de la tendinopatía es muy variable, oscila entre unas horas y 42 días, pudiendo producirse incluso una vez retirado el fármaco $(14,15)$. Es mas frecuente en varones, cualquier tendón puede verse involucrado siendo el mas común el tendón de Aquiles de forma bilateral (4). Su aparición no guarda relación con la dosis del antibiótico, por lo que se considera que depende de la susceptibilidad individual. Los factores de riesgo implicados son la edad superior a 60 años, el tratamiento con corticoides, la insuficiencia renal y cual- 


\section{TABLA I}

TENDINOPATÍA POR QUINOLONAS: CARACTERÍSTICAS CLÍNICAS DE LOS PACIENTES

\begin{tabular}{|c|c|c|c|c|c|}
\hline Pacientes & Caso 1 & Caso 2 & Caso 3 & Caso 4 & Caso 5 \\
\hline Quinolona & levofloxacino & ciprofloxacino & levofloxacino & levofloxacino & ciprofloxacino \\
\hline Edad & 70 & 67 & 73 & 68 & 62 \\
\hline Sexo & varón & mujer & varón & mujer & varón \\
\hline Periodo de latencia* & 72 horas & 48 horas & 48 horas & 6 días & 10 días \\
\hline Localiación & Aquíleo & & & & \\
\hline bilateral & Supraespinoso & & & & \\
\hline bilateral & Aquíleo & & & & \\
\hline bilateral & Aquíleo & & & & \\
\hline derecho & Aquíleo & & & & \\
\hline izquierdo & & & & & \\
\hline F. riesgo & Edad, CE & Edad, IRC & Edad, CE & Edad, CE & Edad \\
\hline Duración* * & 18 días & 10 días & 20 días & 2 meses & 2 meses \\
\hline Evolución & Sin secuelas & Sin secuelas & Sin secuelas & Rotura parcial & Rotura parcial \\
\hline
\end{tabular}

* Inicio de los síntomas tras la introducción del fármaco; **: Duración de los síntomas tras la supresión del fármaco; CE: tratamiento con corticoides; IRC: insuficiencia renal crónica.

quier otra situación que pueda aumentar la fragilidad tendinosa $(4,6,11)$. Algunos autores consideran también como factor de riesgo el antecedente de tendinopatía previa por otra quinolona (4), sugiriendo la existencia de un efecto de clase, que no siempre se demuestra, como sucedió en nuestro segundo caso y en otros referidos recientemente en la literatura (8). Tanto los resultados de laboratorio como la radiología resultan inespecíficos, por lo que el diagnóstico se basa en la exploración física compatible con tendinitis, el antecedente de la toma del fármaco y la mejoría de los síntomas al retirar la medicación. La ecografía (4) y la RM (10) son útiles para mostrar alteraciones precozmente. El tratamiento consiste en reposo, tratamiento sintomático y sobre todo, la retirada de la quinolona, pudiéndose producir roturas tendinosas si no cesa la administración del antibiótico.

Nuestra breve serie de casos (Tabla I) se ajusta a lo ya referido en la literatura, dos fueron mujeres y tres varones, el período de latencia entre la toma del fármaco y la clínica osciló entre dos y diez días, en tres fue el levofloxacino el responsable y en dos el ciprofloxacino. Todos presentaban varios factores de riesgo (edad superior a 60 años, tratamiento con corticoides, IRC). La localización mas frecuente fue en el tendón de Aquiles, en dos pacientes bilateral y en otros dos unilateral. En el caso número dos la localización en el supraespinoso bilateral es menos frecuente, aunque se ha descrito previamente por otros autores (16). A esta paciente se le realizó estudio radiológico de hombros en el que no se demostraba la existencia de calcificaciones ni ningún otro hallazgo. En todos los casos la relación temporal entre el inicio del tratamiento, la aparición de la sintomatología y la respuesta tras la supresión del fármaco descartan razonablemente otras etiologías. La evolución tras el cese de la toma del medicamento y el tratamiento sintomático fue buena en todos los pacientes. La duración de los síntomas desde la retirada del fármaco osciló entre 10 días y 2 meses. Los casos cuatro y cinco presentaron una rotura parcial de fibras, pero en ningún paciente se produjo una rotura tendinosa completa probablemente por la precocidad del diagnóstico.

En suma, queremos subrayar la importancia de considerar la posibilidad de este efecto secundario de la fluoroquinolonas, dado el uso creciente de estos antibióticos tanto en el medio hospitalario como extrahospitalario, siempre que exista una tendinopatía de causa no aclarada, en especial, si existen factores de riesgo de fragilidad tendinosa, ya que sólo la retirada del fármaco puede evitar la rotura del tendón.

\section{Bibliografía}

1. Lipsky BA ,Backer CA. Fluoroquinolone toxicity profiles: A review focusing on newer agentes. Clin Infect Dis 1999; 28: 352-64.

2. Roger RJ, Pierffite C,Netter P: Features of tendon disorders with fluoroquinolnes. Therapie 1994; 49: 75-6.

3. Bailey RS, Kirk JA, Peddie BA: Norfloxacin induced rheumatic disease. NZ Med J 1983; 96: 590.

4. Zabraniecki L, Negrier I, Vergne P, et al. Fluoroquinolone induced tendinopathy: Report of 6 cases. J Rheumatol 1996; 23: 516-9.

5. McEvan Sr; Davey PG: Ciprofloxacin and tenosynovitis. Lancet 1988; 15: 900 .

6. Hernández MV, Peris P, Sierra J, Collado A, Munoz Gómez J. Tendini- tis por fluoroquinolonas. Descripción de dos pacientes. Med Clin (Barc) 1994; 103: 264-6.

7. Ribard P, Audisio F, Kahn MF, et al. Seven achilles tendinitis including 3 complicated by rupture during fluorquinolone therapy: J Rheumatol 1992; 19: 1479-81.

8. Rodriguez-Vera FJ, Pereira Vega A, Pujol de la Llave E. Tendinopatía por quinolonas: tratamiento y efecto de clase en dos nuevos casos. Rev Clin Esp 2004; 204 (1): 35-6.

9. Ribard P, Kanhn MF; Rheumatological side effects of quinolones. Baillere's Clin Rheumatol 1991; 5: 175-91.

10. Pierffite C, Gillet P, Royer RJ. More on fuororoquinolone antibiotiocs 
and tendon rupture. N Eng J Med 1995; 332: 193.

11. Van der Linden PD, Sturkenboom MC, Herings MRC el al. Increased risk of Achilles tendon rupture with quinolone antibacterial use, especially in elderly patients taking oral corticosteroides. Arch Intern Med 2003; 163: 1801-7.

12. Guis S, Bendahan D, Kozak-Ribbens G, et al. Investigation of fluoroquinolone induced mylgia using (31) $\mathrm{P}$ magnetic resonance spestroscopy and in vitro contracture tests. Arthritis Rheum 2002; 46: 774-8.

13. Gómez Rodríguez N, Ibáñez Ruán J, Gónzalez Pérez M. Tendonitis aquílea bilateral y levofloxacino. An Med Interna (Madrid) 2004; 21: 154.

14. Lado Lado FL, Rodríguez Moreno C, Velasco González M, Durán Parrondo C, Moar Calvo B. Rotura parcial bilateral aquílea asociada a levofloxacino. An Med Interna (Madrid) 2005; 22: 28-30

15. Claudio Aros E, Claudio Flores W, Sergio Mezzano A. Tendinitis aqui- liana asociada al uso de levofloxacino: Comunicación de cuatro casos. Rev Med Chil 2002; 130: 1277-81.

16. Casado Burgos E, Viñas Ponce G Lauzurica Valdemoros R, Aleja Olivé Marqués A. Tendinitis por levofloxacino. Med Clin 2000 (Barc); 114: 319.

17. Nuño FJ, Noval J, Suarez M, Guinea O. Dolor aquíleo e impotencia funcional en paciente con enfermedad obstructiva crónica con neumonía. Rev. Clin. Esp 2001; 201: 539-40.

18. Sánchez Muñoz LA, Sanjuán Porugal FJ, Naya Machado J, Castiella Herrero J. Levofloxacino y rotura bilateral del tendón de Aquiles con evolución fatal. An Med Interna (Madrid) 2006; 23: 102.

19. Leone R, Venegoni M, Motola D, Moretti U, Piazzetta V,Cocci A, et al. Adverse drug reactions related to the use of fluoroquinolone antimicrobials. An anlysis of spontaneous reports and fluoroquinolone consumption data from three Italian regions. Drug Saf 2003; 26:109-20. 